\title{
Simulação Computacional para Avaliação de Cenários sobre a Reciclagem de Resíduos Sólidos Urbanos e o seu Impacto na Economia de Energia
}

\author{
Eugênio de Oliveira Simonetto ${ }^{1,2}$, Mauri Leodir Löbler ${ }^{2}$ \\ Universidade Federal de Santa Maria (UFSM) \\ 97.105-090 - Santa Maria - RS - Brasil \\ ${ }^{1}$ UFSM - CTISM - Colégio Técnico Industrial de Santa Maria \\ ${ }^{2}$ UFSM - PPGA - Programa de Pós-Graduação em Administração \\ \{eosimonetto,mllobler\}@gmail.com
}

\begin{abstract}
In this article is presented a computer simulation model, since their development until its validation, which aims to support environmental managers in their decisions regarding the definition and / or maintenance of solid waste policies recycling, as well as evaluating the benefits of process in the environment (in this article we evaluated the energy savings). For the model development was considered: the rate of natural population growth, percentage of solid waste recycled, gravimetric composition of the material in the total waste generated, the amount of waste generated per inhabitant and energy savings caused by each distinct type of material. Through the model results generated, end users (environmental managers) thereof may, for example, set incentives to reduce the total generation of solid waste and to assess the relative benefits of electrical energy savings caused by recycling.
\end{abstract}

Resumo. Nesse artigo apresenta-se um modelo de simulação computacional que tem por objetivo auxiliar gestores ambientais em suas decisões com relação à definição elou manutenção de políticas de reciclagem de resíduos sólidos, bem como avaliar os benefícios do processo no meio ambiente (nesse artigo foi avaliada a economia de energia elétrica). Para a construção do modelo considerou-se: a taxa de crescimento natural populacional, percentual de resíduo sólido reciclado, composição gravimétrica no total de resíduo gerado, quantidade de resíduos gerada e a economia de energia elétrica ocasionada por cada tipo distinto de material. Através dos resultados gerados pelo modelo gestores poderão, por exemplo, definir incentivos à redução da geração total de resíduos sólidos e avaliar os benefícios relativos à economia de energia elétrica ocasionados pela reciclagem.

\section{Introdução}

A evolução da gestão integrada dos resíduos sólidos nos últimos anos transformou-a em uma importante, e crítica, área para os gestores ambientais, municipais e população em geral. Toda esta evolução ocasionou, também, novos problemas a serem tratados, tais como problemas ambientais, econômicos e legais. Dentre os problemas, encontram-se os decorrentes da má utilização e destinação dos resíduos, os quais podem causar grandes impactos desfavoráveis para o meio ambiente, o que caracteriza um desperdício da matéria originalmente utilizada. 
Para que os resíduos gerados pela população e pelas indústrias venham a ser reaproveitados e, contribuam para a redução do uso de recursos naturais que se tornam cada vez mais escassos, é fundamental que se tenham informações precisas e confiáveis sobre as características físico-químicas dos resíduos, quantidades geradas, coletadas e o destino dado aos mesmos. A reciclagem de resíduos é uma excelente alternativa para a busca do desenvolvimento sustentável na gestão integrada dos resíduos sólidos, pois permite a economia de energia elétrica, bem como um menor uso de recursos naturais na produção de aço, alumínio, papel / papelão, plástico e vidro. Outro aspecto que denota a importância da reciclagem de resíduos é o fato de ser considerada uma ação prioritária pela Política Nacional de Resíduos Sólidos - PNRS [Ministério... 2011].

O estudo da gestão integrada de resíduos sólidos tem atraído a atenção de pesquisadores da área de sistemas de apoio a decisão nos últimos anos, pois através do uso das ferramentas dessa área pode-se representar uma situação do mundo real, estudar seu comportamento e tomar decisões com base nas conclusões extraídas [Bani et al. 2009], [Simonetto e Borenstein 2006]. Prova disso, são os inúmeros trabalhos publicados na área, os quais vão desde a alocação de veículos para a coleta dos resíduos [Everett and Shahi 1997] até a construção de modelos de avaliação de impacto ambiental provocado pela disposição final dos resíduos, levando em consideração o tipo de disposição, o tipo de resíduo e a área onde o mesmo foi depositado [Perrodin et al. 2002]. Diversos outros autores [Bani et al. 2009] já utilizaram técnicas e métodos de sistemas de apoio a decisão para desenvolver estudos nesta área específica, embora bastante escassos no Brasil.

O objetivo do artigo é apresentar a modelagem, desenvolvimento e validação de um modelo de simulação computacional, que permite aos decisores da área ambiental avaliarem e analisarem cenários acerca da reciclagem de resíduos de sólidos, tanto no que tange à quantidade de resíduos reciclados, como nos benefícios gerados pela mesma. No caso do artigo, foi avaliada a economia de energia elétrica obtida com o processo de reciclagem dos diferentes tipos de materiais reciclados. O problema de pesquisa consistiu da investigação, definição e validação das variáveis componentes do modelo de simulação, bem como da concepção e validação do mesmo. Para o desenvolvimento do modelo computacional foram utilizadas técnicas oriundas da área de system dynamics [Daellenbach e McNickle 2005], [Gharajedaghi 2006]. O uso de ferramentas da área de sistemas de apoio a decisão busca agregar qualidade ao processo decisório, pois, ainda hoje, muitas decisões sobre a gestão dos resíduos sólidos embasadas, somente, na experiência dos gestores [Chang e Wei 2000].

Um claro exemplo da economia de energia elétrica ocasionada pela reciclagem é o caso do alumínio [Hisatugo e Marçal Jr. 2007], onde para produção de 1 tonelada a partir da matéria prima utiliza-se 17,6 Mwh e, para a produção da mesma quantidade, a partir do alumínio reciclado, utiliza-se apenas 0,75 Mwh gerando, assim, uma economia de 16,85 Mwh (95\% de economia de energia elétrica).

O artigo está organizado da seguinte forma: Na seção 2 é apresentada a metodologia de pesquisa utilizada para o desenvolvimento do estudo. Na seção 3 são descritos o problema de modelagem, as variáveis componentes e o modelo desenvolvido. Na seção 4 são apresentados a validação, os cenários de simulação, o experimento utilizando o modelo, bem como a discussão de resultados. As considerações finais são apresentadas na seção 5.

\section{Método de Pesquisa}


Nesse trabalho o método de pesquisa adotado para o desenvolvimento do modelo computacional foi baseado na metodologia proposta por Law e Kelton (1991) e, consistiu das seguintes etapas: (1) estudos exploratórios em artigos científicos, manuais de referência e entrevistas com gestores da área de resíduos sólidos, na qual o problema foi caracterizado e estruturado, bem como foi formulada a hipótese dinâmica, a qual é apresentada na seção 2.3; (2) desenvolvimento da solução, pela construção de modelos formais capazes de representar o problema; (3) implementação computacional da solução, utilizando-se o simulador Vensim [Vensim 2012] da área de system dynamics; (4) validação da solução, através de testes em laboratório e em campo, para verificar se os resultados obtidos estão de acordo com a realidade observada, bem como através da simulação de um experimento utilizando três cenários para tal.

Os cenários que foram utilizados para a validação do modelo foram gerados a partir de análises, onde foram utilizados dados históricos relativos aos censos populacionais de 2000 e 2010 [IBGE 2010], o Panorama dos Resíduos Sólidos no Brasil-2010 [ABRELPE 2012], o Plano Nacional de Resíduos Sólidos [Ministério 2011], bem como do Diagnóstico de Manejo de Resíduos Sólidos [Ministério 2010] e, também, através da participação de pesquisadores e especialistas na área. Os conceitos envolvidos no desenvolvimento da pesquisa (system dynamics e reciclagem de resíduos) são apresentados a seguir nas seções 2.1 e 2.2.

\subsection{System Dynamics}

A metodologia system dynamics (SD) permite o estudo do comportamento dos sistemas ao longo do tempo, de maneira a permitir a avaliação das consequências de nossas decisões [Daellenbach e McNickle 2005]. Por tal motivo e pela necessidade de estudar a reciclagem dos resíduos em um horizonte temporal futuro decidiu-se utilizá-la na modelagem e simulação computacional. Um modelo de SD pode ser interpretado como a estrutura resultante da interação de políticas. Esta estrutura é formada por dois componentes principais, que são os estoques e os fluxos, tanto que Ford (2009) define os SD como uma combinação de estoques e fluxos que utilizam uma estrutura computacional para serem simulados. Os estoques retratam as variáveis que são acumuladas e os fluxos as funções de decisão ou políticas de um sistema. Estes componentes podem estar organizados na forma de relações circulares de causa e efeito, conhecidas como feedback de balanço ou de reforço, e estão sujeitos à defasagens de tempo no sistema. Sufian e Bala (2007) utilizaram essa abordagem para a modelagem do sistema de gestão de resíduos sólidos da cidade de Dhaka-Bangladesh.

\subsection{Reciclagem de Resíduos}

Reciclagem, segundo O'Leary (1999), é o processo pelo qual, resíduos que são destinados à disposição final são coletados, processados e reutilizados. Monteiro (2001) define reciclagem como sendo a separação de materiais do lixo domiciliar, tais como papéis, plásticos, vidros e materiais, com a finalidade de trazê-los de volta à indústria para serem beneficiados. Estes materiais são novamente transformados em produtos comercializáveis.

A reciclagem dos resíduos sólidos é uma excelente alternativa para propiciar a preservação de recursos naturais, a economia de energia, redução de área que demanda o aterro sanitário, geração de emprego e renda, assim como a conscientização da população para questões ambientais. Porém, para um melhor funcionamento, é de suma importância que se implante nas cidades um amplo sistema de coleta seletiva, onde os RSU potencialmente recicláveis sejam segregados nas residências e coletados pelo sistema municipal de coleta seletiva. Apesar de ser uma boa alternativa para a redução de resíduos destinados aos aterros, 
apenas uma pequena parcela (aproximadamente 10\%) dos resíduos são reutilizados ou reciclados nas cidades gaúchas, segundo a CEMPRE (Organização Não-Governamental Compromisso Empresarial para Reciclagem) [Netto 2001].

\section{Modelo de Simulação e Variáveis Componentes}

A Política Nacional de Resíduos Sólidos (PNRS) [Ministério 2011] definiu a ordem de ações a ser seguida na gestão de resíduos, onde, dentre as quais, foi incluída a reciclagem como uma das principais ações. A reciclagem, nos termos da lei, é o processo de transformação dos resíduos envolvendo a alteração de suas propriedades físicas, físico-químicas ou biológicas, visando à transformação desses em insumos ou novos produtos. [ABRELPE 2012]

Associado à prioridade atribuída pela PNRS, verifica-se a crescente geração de resíduos sólidos pela população [ABRELPE 2012], logo, demandando que alternativas viáveis ao melhor aproveitamento dos resíduos sólidos sejam criadas e executadas. Nesse aspecto, a reciclagem dos resíduos surge como uma alternativa viável, já que no momento em que o material é reciclado ele não demanda espaço em um aterro sanitário, não polui o meio ambiente e não utiliza recursos naturais em excesso na sua transformação.

Tendo por embasamento a prioridade atribuída à reciclagem pela PNRS e os ganhos ambientais ocasionados por essa, nesse trabalho buscou-se o desenvolvimento de um modelo de simulação o qual permitisse tanto aos gestores ambientais, quanto os da área de resíduos sólidos avaliar políticas de reciclagem de resíduos no que refere-se aos materiais reciclados e aos ganhos ambientais, visando o desenvolvimento sustentável, gerados por esta. No modelo desenvolvido os materiais reciclados considerados foram o alumínio, aço, papel / papelão, vidro e plástico. Quanto à questão referente aos ganhos ambientais, no modelo, foi avaliada a economia de energia elétrica gerada pela reciclagem de tais materiais. Outros recursos poderiam ser avaliados, tais como: redução do corte de árvores, redução do consumo de água, minimização do uso de petróleo, etc.

As decisões, a partir das análises geradas pelo modelo, poderão envolver a busca pela elevação da taxa de reciclagem (com campanhas de conscientização da população), busca pela redução do consumo, incentivos ao aumento do "consumo verde" (reduzindo resíduos orgânicos no meio ambiente) [Mansvelt 2010], bem como outras análises e observações de interesse dos gestores ambientais e/ou municipais, desde que as mesmas sejam exequíveis no modelo de simulação. O modelo foi implementado buscando simplificar a interação usuáriocomputador, para que análises do tipo "o que se?" (what-if?), comuns em modelos de simulação, sejam de rápida e simples execução.

Para a definição das variáveis do modelo de simulação (Figura 1) foram utilizados trabalhos acadêmicos e governamentais da área de resíduos sólidos [ABRELPE 2012], [IBGE 2010], [Ministério 2010], [Ministério 2011], [Monteiro et al. 2001], [UNEP 2005]. A validação dessas foi executada com a participação de gestores ambientais e profissionais da área de resíduos sólidos. As variáveis selecionadas, bem como suas inter-relações com outras variáveis, as quais influenciam nos valores totais de geração e disposição final dos resíduos sólidos urbanos, são:

- A taxa de nascimento anual (NascTaxa), a taxa de mortalidade anual (MortTaxa), a taxa de imigração anual (ImigTaxa) e a taxa de emigração anual (EmigTaxa), todas estas influenciando diretamente os fluxos de entrada e saída populacional (AcrescPop $\boldsymbol{e}$ DecrPop), os quais determinam a população total (Populacao) do município. Foi utilizada no 
modelo a taxa de crescimento natural ou vegetativo (total de nascimentos - total de mortes) a qual corresponde à única forma possível de crescimento ou redução da população mundial e, quando se analisa o crescimento de áreas específicas devem ser consideradas, também, as migrações. Essas variáveis são representadas pelas equações (1), (2) e (3) na formulação matemática do modelo de equações diferenciais ordinárias. (Figura 2)

- a quantidade média de resíduos (RSUPerCapita) gerada por cada habitante multiplicada pela população total do município resulta na quantidade total de resíduos (GeracaoRSU) do município. As variáveis descritas são representadas na equação (4) do modelo matemático da Figura 2;

- os fluxos de entrada de resíduos recicláveis nos diferentes tipos de material reciclável (AcoRecic, AluminioRecic, PapelRecic, PlasticoRecic, VidroRecic), os quais representam a totalidade anual de resíduos sólidos recicláveis de cada um dos diferentes tipos de material e, são obtidos através do produto da quantidade total de resíduos (GeracaoRSU) pela taxa percentual de participação do resíduo sólido no total de resíduos gerados (composição gravimétrica) e, ainda, o produto desse pela taxa de reciclagem do material em análise. As equações (Figura 2) que representam as variáveis relativas aos fluxos de entrada estão descritas no modelo matemático pelas equações (5), (6), (7), (8) e (9);

- a quantidade total acumulada de cada tipo de material reciclado é representada pelas variáveis de nível Aco (aço), Aluminio (alumínio), Papel (papel/papelão), Plastico (plástico) e Vidro (vidro). As variáveis relativas aos totais acumulados de resíduos recicláveis estão descritas nas equações (10), (11), (12), (13) e (14) na Figura 2;

- a quantidade de energia elétrica economizada (EconomiaEnergia) com o processo de reciclagem é obtida através da equação (15) descrita na Figura 2, a qual para seu cálculo utiliza dados referentes à economia obtida pelos distintos tipos de materiais, bem como a geração total de cada um destes.

O modelo de simulação utilizando Systems Dynamics (Figura 1) e sua formulação matemática (Figura 2) auxiliam-nos na compreensão das variáveis e de suas inter-relações, facilitando assim o entendimento do mesmo.

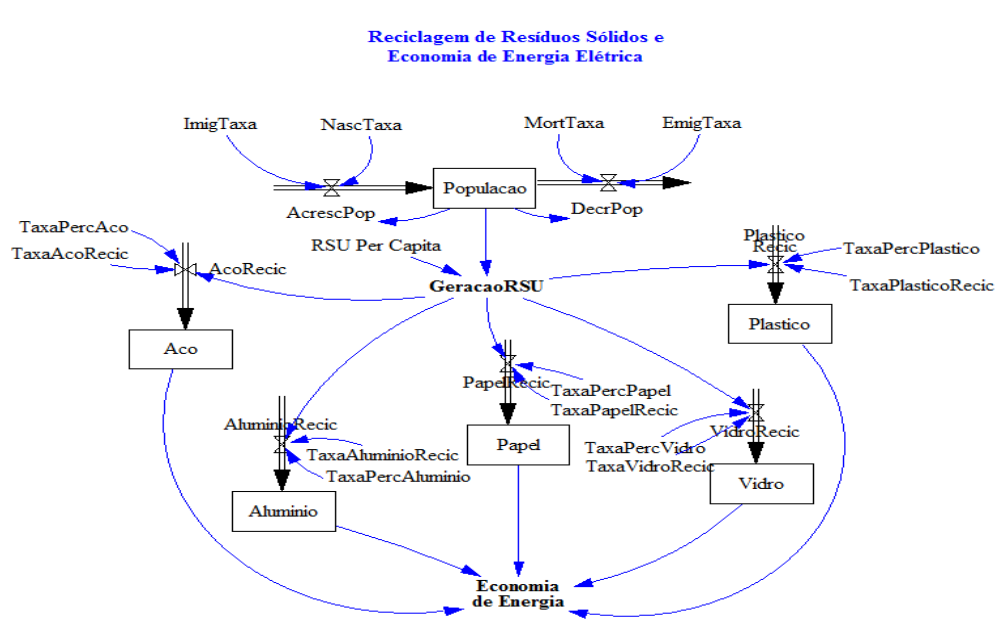

Figura 1. Modelo de simulação desenvolvido 


\section{Validação e Experimentos no Modelo de Simulação}

Para o desenvolvimento da validação do modelo de simulação foi utilizada a abordagem proposta por Finlay (1994) para validações de modelos decisórios e sistemas de apoio a decisão (SAD). A abordagem de Finlay (1994) propõe a validação de modelos decisórios através da combinação de dois métodos:

Validação Analítica - Cada parte do modelo decisório é verificada individualmente e, também, a sua integração com as outras partes componentes do sistema.

Validação Sinótica - Neste tipo de validação é analisado o modelo decisório como um todo e, então a execução do sistema é testada. A validação é executada através da comparação dos resultados do mundo real com os resultados (saídas) produzidos pelo modelo. Se o sistema produz resultados "aceitáveis", de acordo com as entradas fornecidas, podemos dizer que o sistema é válido.

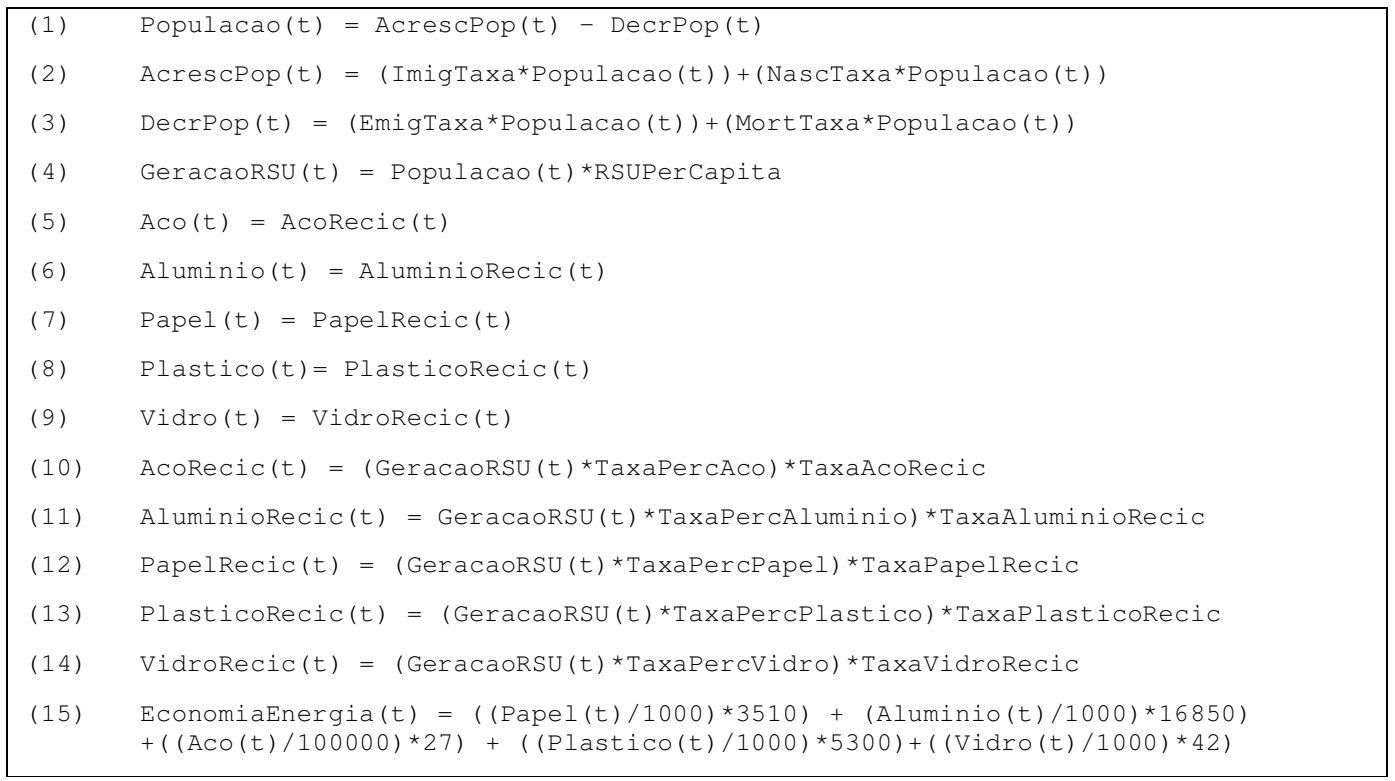

Figura 2. Formulação Matemática do modelo de simulação desenvolvido

Na primeira fase de validação, ou seja, da validação conceitual do problema, são validados os requisitos funcionais, não funcionais e as variáveis componentes do modelo. Neste momento inicial, foram utilizados dados de artigos científicos, da Política Nacional de Resíduos Sólidos [Ministério 2011], de manuais técnicos referentes à área de resíduos sólidos e, contou com a participação de especialistas em gestão ambiental. Na segunda fase de validação, quando da implementação no simulador Vensim [Vensim 2012], foram utilizados dados históricos sobre a população brasileira e gestão de RSU para a verificação dos módulos individualmente e sua integração com os demais módulos componentes do modelo (validação analítica). Foram, também, verificados e validados os resultados gerados pelo modelo de simulação e sua conformidade com dados e resultados obtidos no mundo real, denotando a corretude do modelo (validação sinótica). Em ambas as fases os resultados atenderam às expectativas dos projetistas da simulação e especialistas (gestores ambientais).

$\mathrm{Na}$ etapa de validação posterior, para a construção do experimento, foram utilizados dados e taxas reais (população, crescimento populacional e dados referentes à gestão dos 
resíduos sólidos) de um município do Rio Grande do Sul. Para tal, foram gerados 2 (dois) cenários a serem simulados no modelo: (a) Cenário atual com variação atual das taxas e; (b) cenário positivo, o qual é baseado em taxas crescentes da reciclagem de resíduos, bem como da manutenção estática da taxa de geração de resíduos sólidos por parte da população. Esse tipo de validação pode-se reconhecer como análise de sensibilidade do modelo de simulação, visto que foram utilizadas variáveis controladas nos inputs e verificadas as variações nos resultados. O detalhamento e quantificação de taxas para cada cenário simulado são apresentados a seguir na subseção 4.1.

\subsection{Cenários simulados no modelo}

Para melhor descrever os cenários simulados, na Tabela 1 serão apresentadas as taxas básicas referentes ao crescimento populacional, à geração de resíduos, à composição gravimétrica dos materiais reciclados no total de resíduo gerado e a taxa de reciclagem de cada material analisado pelo modelo. Para obtenção de tais dados foram analisados o Censo Populacional [IBGE 2010], o Panorama dos Resíduos Sólidos no Brasil-2011 [ABRELPE 2012] e a Política Nacional de Resíduos Sólidos [Ministério 2011]. Os cenários gerados foram concebidos pelos pesquisadores, com o auxílio de gestores ambientais.

Tabela 1. Taxas básicas consideradas na simulação

\begin{tabular}{|c|c|}
\hline Crescimento Populacional & $\begin{array}{l}\text { Foram consideradas as taxas atuais de natalidade e mortalidade, onde } \\
\text { o crescimento populacional médio de um município do Rio Grande } \\
\text { do Sul foi de } 0,7 \% \text { [IBGE 2010]. }\end{array}$ \\
\hline Geração de Resíduos & $\begin{array}{l}\text { A taxa média de geração de resíduos urbanos diária é de, } \\
\text { aproximadamente, } 1,223 \mathrm{~kg} / \text { pessoa [ABRELPE 2012]. A evolução da } \\
\text { geração anual é de } 2 \% \text {. }\end{array}$ \\
\hline $\begin{array}{l}\text { Composição Gravimétrica do } \\
\text { Material nos Resíduos } \\
\text { Gerados }\end{array}$ & $\begin{array}{l}\text { As taxas foram obtidas em [Ministério 2011]: } \\
\text { Aço - corresponde a 2,3\% dos resíduos sólidos gerados. } \\
\text { Alumínio - corresponde a } 0,6 \% \text { dos resíduos sólidos gerados. } \\
\text { Papel / Papelão - corresponde a } 13,1 \% \text { dos resíduos sólidos gerados. } \\
\text { Plástico- corresponde a } 19 \% \text { dos resíduos sólidos gerados. } \\
\text { Vidro - corresponde a } 2,4 \% \text { dos resíduos sólidos gerados. }\end{array}$ \\
\hline $\begin{array}{l}\text { Taxa de Reciclagem do } \\
\text { Material }\end{array}$ & $\begin{array}{l}\text { As taxas foram obtidas em [Ministério 2011]: } \\
\text { Aço - 35\% do aço coletado são reciclados. } \\
\text { Alumínio - 36\% do alumínio coletado são reciclados. } \\
\text { Papel / Papelão - } 43 \% \text { do papel coletado são reciclados. } \\
\text { Plástico- } 19 \% \text { do plástico coletado são reciclados. } \\
\text { Vidro - } 21 \% \text { do vidro coletado são reciclados. }\end{array}$ \\
\hline $\begin{array}{l}\text { Economia de Energia Gerada } \\
\text { pela Reciclagem }\end{array}$ & $\begin{array}{l}\text { As taxas foram obtidas em [Garbelini et al. 2011], [Hisatugo e Marçal } \\
\text { Jr. 2007], [Waste Management 2012]: } \\
\text { Aço - } 27 \text { Kwh /100 t. } \\
\text { Alumínio - } 16850 \mathrm{Kwh} / \mathrm{t} \text {. } \\
\text { Papel / Papelão }-3510 \mathrm{Kwh} / \mathrm{t} \text {. } \\
\text { Plástico - } 5300 \mathrm{KWh} / \mathrm{t} \text {. } \\
\text { Vidro - } 42 \mathrm{Kwh} / \mathrm{t} \text {. }\end{array}$ \\
\hline
\end{tabular}

Cenário Positivo - Na concepção do cenário positivo foram simuladas taxas crescentes da reciclagem dos materiais analisados e de sua composição gravimétrica no total de resíduos gerados, em contrapartida, manteve-se estática a taxa de geração per capita de resíduos sólidos. O crescimento populacional foi mantido para fins desse cenário simulado. A descrição do cenário pode ser visualizada na Tabela 2. 
Cenário Atual da Reciclagem - No cenário atual da reciclagem foram consideradas as taxas de crescimento populacional e de geração de resíduos per capita, porém manteve-se estática a composição gravimétrica e, considerou-se um pequeno aumento nos índices de reciclagem do material. O detalhamento do cenário pode ser visualizado na Tabela 2.

Tabela 2. Taxas utilizadas na simulação

\begin{tabular}{|l|l|l|}
\hline \multicolumn{1}{|c|}{ Variável } & \multicolumn{1}{|c|}{ Cenário Atual } & \multicolumn{1}{c|}{ Cenário Positivo } \\
\hline $\begin{array}{l}\text { Crescimento } \\
\text { Populacional }\end{array}$ & $\begin{array}{l}\text { Foram consideradas as taxas atuais de } \\
\text { natalidade e mortalidade do município } \\
\text { analisado. }\end{array}$ & $\begin{array}{l}\text { Foram consideradas as taxas atuais de } \\
\text { natalidade e mortalidade do município } \\
\text { analisado. }\end{array}$ \\
\hline Geração de Resíduos & $\begin{array}{l}\text { Nesse cenário a taxa de geração } \\
\text { considerou-se a evolução anual de 2\%. }\end{array}$ & $\begin{array}{l}\text { Nesse cenário a taxa de geração } \\
\text { permaneceu estática até o fim do } \\
\text { tempo simulado e não foi considerada } \\
\text { a evolução anual de 2\%. }\end{array}$ \\
\hline $\begin{array}{l}\text { Composição } \\
\text { Material nos } \\
\text { Resíduos Gerados }\end{array}$ & $\begin{array}{l}\text { Nesse cenário inalterou-se o índice de de } \\
\text { composição gravimétrica dos materiais } \\
\text { reciclados com relação à geração de } \\
\text { resíduos sólidos. }\end{array}$ & $\begin{array}{l}\text { Nesse cenário foi considerado um } \\
\text { aumento de 30\% da composição } \\
\text { gravimétrica para os dez últimos anos } \\
\text { simulados (20 ao 30 }) .\end{array}$ \\
\hline $\begin{array}{l}\text { Taxa de Reciclagem } \\
\text { do Material }\end{array}$ & $\begin{array}{l}\text { Nesse cenário, considerou-se um } \\
\text { aumento de 0,3\% nas taxas de } \\
\text { reciclagem (ao ano), para cada tipo de } \\
\text { material reciclado. }\end{array}$ & $\begin{array}{l}\text { Nesse cenário, nos primeiros dez anos } \\
\text { simulados considerou-se que houve um } \\
\text { crescimento de 30\% na taxa de } \\
\text { reciclagem para cada um dos materiais. } \\
\text { Do 11 } \\
\text { um ano ao 30 ano considerou-se } \\
\text { aumento de 50\% na taxa de } \\
\text { reciclagem, para cada tipo de material. }\end{array}$ \\
\hline
\end{tabular}

\subsection{Experimento}

Definidos os cenários para a realização do experimento com o uso do modelo foram executadas as simulações. Conforme descrito anteriormente, os dados utilizados em ambos cenários foram de um município com, aproximadamente, 265 mil habitantes do interior do Rio Grande do Sul, o qual possui coleta seletiva de resíduos sólidos, bem como unidades de triagem de resíduos sólidos visando à reciclagem dos mesmos. As outras taxas foram obtidas em documentos técnicos, conforme pode ser visualizado anteriormente na seção 4.1. O horizonte de tempo simulado no experimento foi de 30 (trinta) anos, porém a configuração dessa variável fica a cargo dos gestores (possíveis usuários finais) e/ou projetista da simulação.

$\mathrm{Na}$ execução das simulações foi utilizado o simulador Vensim [Vensim 2012] em uma estrutura computacional com processador Intel Core (i5 2450) de 2,5 Ghz, 4 Gb de memória RAM e o tempo de execução da simulação dos dois cenários foi na ordem de milionésimos de segundo. Os resultados obtidos com o uso do modelo são apresentados a seguir na subseção 4.3.

\subsection{Resultados Obtidos}

Neste artigo serão analisados os resultados referentes à quantidade de resíduos reciclada com relação à geração total de resíduos sólidos, bem como os relacionados à economia de energia elétrica gerada pela reciclagem de resíduos.

Primeiramente, analisar-se-á a quantidade de resíduos reciclada em ambos os cenários, para tal foi considerado o último ano simulado no modelo, ou seja, o $30^{\circ}$ ano. Nessa análise verifica-se o alto de índice de reciclagem ocasionado pelo cenário positivo, onde o mesmo atinge a marca de $27,33 \%$ de reciclagem dos resíduos gerados (42.670 toneladas). Nessa 
mesma análise, o cenário atual atinge o índice de $11,76 \%$ de reciclagem dos resíduos gerados (24503 toneladas), o que denota um crescimento de 9,3\% ao índice de reciclagem dos dias atuais (aproximadamente 10,7\%). Os resultados gerados, bem como o detalhamento da quantidade simulada para cada resíduo individualmente pode ser visualizado na Tabela 3.

Tabela 3. Resultados dos cenários no último ano simulado e relação ao total de resíduos gerado

\begin{tabular}{|c|c|c|c|c|}
\hline & \multicolumn{2}{|c|}{ Cenário Atual (Ano 30) } & \multicolumn{2}{c|}{ Cenário Positivo (Ano 30) } \\
\hline Material & $\begin{array}{c}\text { Quantidade } \\
\text { Reciclada }\end{array}$ & $\begin{array}{c}\text { Relação } \\
\text { Geração } \\
\text { Total }\end{array}$ & $\begin{array}{c}\text { Quantidade } \\
\text { Reciclada }\end{array}$ & $\begin{array}{c}\text { Relação } \\
\text { Geração } \\
\text { Total }\end{array}$ \\
\hline Aço & $1.830 .390 \mathrm{~kg}$ & $0,88 \%$ & $3.196 .550 \mathrm{~kg}$ & $2,05 \%$ \\
\hline Alumínio & $489.992 \mathrm{~kg}$ & $0,24 \%$ & $876.769 \mathrm{~kg}$ & $0,56 \%$ \\
\hline Papel / Papelão & $12.799 .600 \mathrm{~kg}$ & $6,14 \%$ & $22.293 .400 \mathrm{~kg}$ & $14,28 \%$ \\
\hline Plástico & $8.233 .200 \mathrm{~kg}$ & $3,95 \%$ & $14.306 .400 \mathrm{~kg}$ & $9,16 \%$ \\
\hline Vidro & $1.149 .980 \mathrm{~kg}$ & $0,55 \%$ & $1.997 .090 \mathrm{~kg}$ & $1,28 \%$ \\
\hline TOTAL & $\mathbf{2 4 . 5 0 3 . 1 6 2} \mathbf{~ k g}$ & $\mathbf{1 1 , 7 6 \%}$ & $\mathbf{4 2 . 6 7 0 . 2 0 9} \mathbf{~ k g}$ & $\mathbf{2 7 , 3 3 \%}$ \\
\hline
\end{tabular}

O cenário positivo com um índice de reciclagem de $27,33 \%$ apresenta um excelente crescimento de $153 \%$ se relacionado ao índice de reciclagem atual, porém com todo esse crescimento, o índice ainda é menor que o registrado em muitos países europeus. Por exemplo, a Holanda apresenta um percentual de $32 \%$ de reciclagem dos resíduos [EUROSTAT 2011]. Para demonstrar o quão pequena é a quantidade de resíduos reciclados no Brasil é apresentado na Figura 3 um gráfico comparativo entre a reciclagem de papel / papelão (resíduo de maior índice de reciclagem) com a geração total de resíduos em todo o período simulado. Ao final do tempo simulado, no cenário positivo, o índice de reciclagem do papel foi de 14,28\% (22.203 toneladas), enquanto no cenário atual o índice de reciclagem foi de 6,14\% (12.800 toneladas).

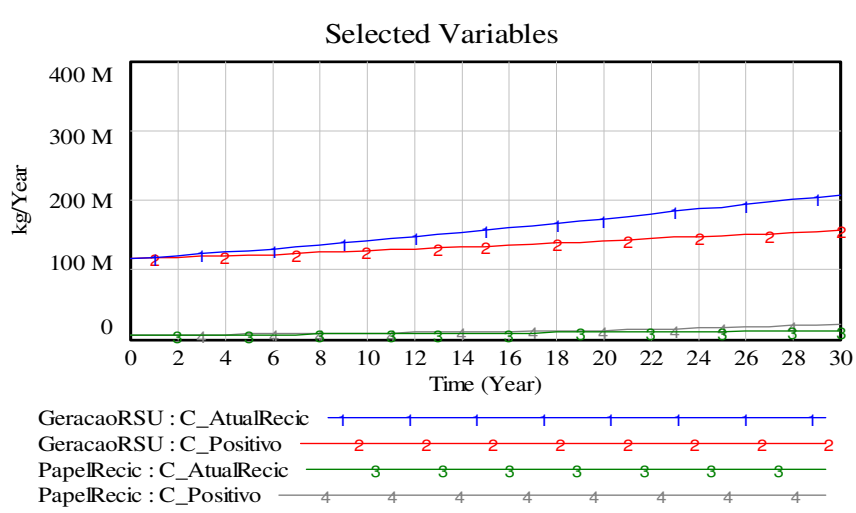

Figura 3. Relação entre a quantidade de papel reciclado e o total de resíduos sólidos gerado

Uma outra análise executada no modelo de simulação foi a da quantidade de energia elétrica economizada ao longo dos trinta anos simulados, de acordo com os índices apresentados em [Garbelini et al. 2011], [Hisatugo e Marçal Jr. 2007], [Waste Management 2012] para cada material analisado. Nessa análise tornam-se evidentes os benefícios da reciclagem de resíduos sólidos ao meio ambiente e à preservação dos recursos naturais, por exemplo, no cenário positivo obtém-se uma economia mensal média de $7500 \mathrm{Mwh}$ e, no cenário atual, uma economia mensal de $5800 \mathrm{Mwh}$. Tais valores são considerados excelentes, pois o consumo aproximado total de uma cidade do porte da analisada é de $82000 \mathrm{Mwh} / \mathrm{mês}$ 
[CEEE 2012], assim sendo, estima-se no cenário positivo uma economia de energia elétrica de $9,1 \%$ ao mês e, no cenário atual uma economia de $7 \%$. Na Figura 4 apresenta-se o total acumulado de energia elétrica nos anos simulados, onde verifica-se que no cenário positivo o valor é de 2.694.280 Mwh e no cenário atual de 2.108.060 Mwh.

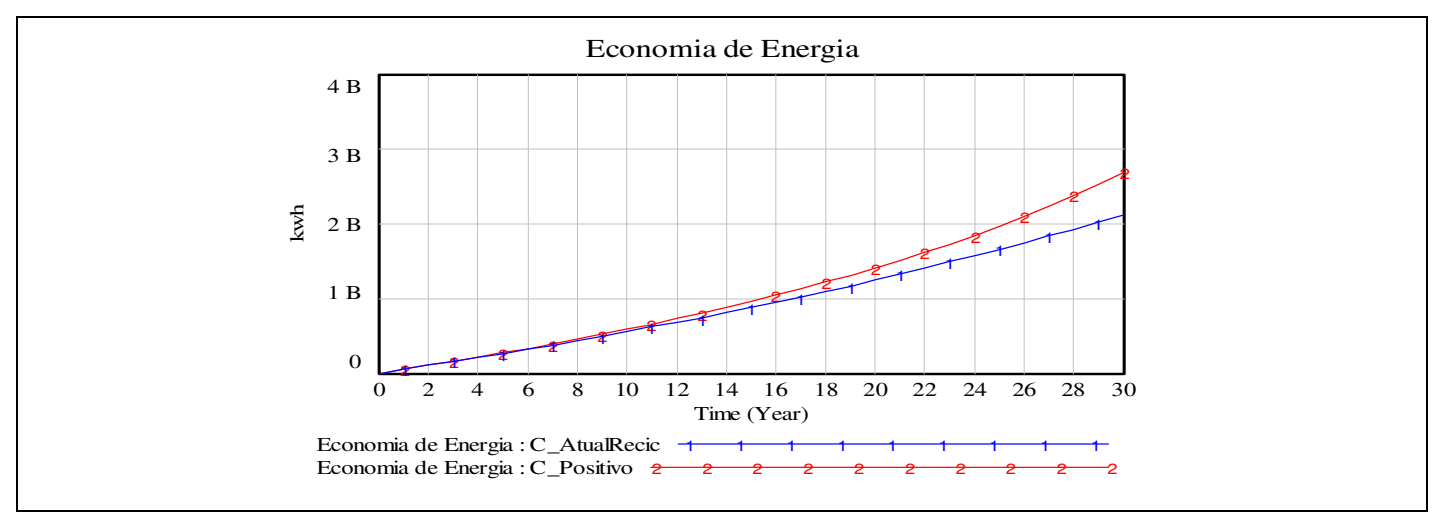

Figura 4. Economia de energia elétrica acumulada ao longo de $\mathbf{3 0}$ anos.

\section{Considerações Finais}

O objetivo do artigo foi o de apresentar o desenvolvimento, a validação e utilização de um modelo de simulação computacional para auxílio dos gestores ambientais no processo decisório, às políticas referentes à reciclagem de resíduos sólidos. Para o desenvolvimento do modelo foram utilizadas variáveis, tais como, a taxa de crescimento natural populacional (nascimentos e mortes), percentual de resíduo sólido reciclado (para cada tipo de material), composição gravimétrica do material no total de resíduos gerados, quantidade de resíduos gerada por habitante e a economia de energia elétrica ocasionada por cada tipo distinto de material em um determinado período de tempo (que, no modelo desenvolvido, pode variar de minutos a anos).

Através dos resultados gerados pelo modelo, os usuários finais (gestores ambientais) do mesmo poderão, por exemplo, definir incentivos à redução da geração total de resíduos sólidos, incentivos ao aumento das taxas de consumo verde, produzir campanhas valorizando o reuso e a reciclagem de materiais e avaliar os benefícios relativos à economia de energia elétrica ocasionados pela reciclagem.

No artigo foram apresentados dois cenários, nos quais o modelo foi verificado e validado utilizando dados de um município do Rio Grande do Sul. Os resultados gerados foram apresentados aos gestores ambientais, os quais testaram o modelo novamente e satisfizeram-se com o mesmo. Os cenários apresentados no artigo foram gerados exclusivamente para a validação do modelo, porém o mesmo pode ser configurado conforme a necessidade do usuário final, tendo em vista o modelo ser aberto e reconfigurável.

Com relação aos resultados produzidos pelo modelo, destaca-se o cenário positivo, pois o mesmo buscou retratar um perfil de população preocupada com as questões ambientais, ou seja, gerando menos resíduo sólido, aumentando a quantidade de resíduo potencialmente reciclável e as taxas de reciclagem. Nessa análise, a quantidade de resíduos reciclados teria um acréscimo de $153 \%$ com relação aos padrões vigentes. Quanto ao cenário atual, a quantidade de resíduos reciclados, ao final do tempo simulado, teria um crescimento de 9,3\%. Apesar de todo o crescimento ocasionado pelo cenário positivo, com um índice de reciclagem de 
aproximadamente $27 \%$, o mesmo está bem abaixo de países da comunidade europeia, tais como, Alemanha, Bélgica, Suécia, Holanda e Irlanda, os quais apresentam índices superiores a $30 \%$ de reciclagem dos resíduos sólidos gerados.

Quanto à economia de energia elétrica, apesar do cenário positivo gerar uma economia superior (7500 Mwh/mês), os padrões atuais (cenário atual) apresentam uma considerável possibilidade de redução de $5800 \mathrm{Mwh} / \mathrm{mês}$, o que denota a vital importância da reciclagem de resíduos sólidos na busca pelo desenvolvimento sustentável e na Política Nacional de Resíduos Sólidos.

Como trabalho futuro pretende-se avaliar, junto à reciclagem, o reuso de material descartado, bem como a compostagem de resíduos. Por fim, ressalta-se que o modelo, após ser avaliado por gestores da área ambiental, satisfez a necessidade de informação dos mesmos.

\section{Referências Bibliográficas}

ABRELPE - Associação Brasileira de Empresas de Limpeza Pública e Resíduos Especiais (2012). Panorama dos Resíduos Sólidos no Brasil-2012. ABRELPE.

Bani, M.S. et al (2009). The Development of Decision Support System for Waste Management; a Review. World Academy of Science, Engineering and Technology, v.49, páginas 161-168.

CEEE - Companhia Estadual de Energia Elétrica (2012). Balanço Energético de 2011. CEEE, http://www.ceee.com.br/pportal/ceee/component/Controller.aspx?CC=46490.Agosto.

Chang, N., Parvathinathan,G. and Breeden, J.B. (2008). Combining GIS with fuzzy multicriteria decision-making for landfill sitting in a fast growing urban region, Journal of Environmental Management, v.87, p.139-153.

Chang, N.; Wei, Y. (2000). Siting recycling drop-off in urban area by genetic algorithm-based fuzzy multiobjective nonlinear integer programming modeling. Fuzzy Sets and Systems, v.114, páginas 133-149.

Daellenbach, H.G. and McNickle, D.C. (2005). Decision making through systems thinking. Palgrave Macmillan.

EUROSTAT- European Commission Statistical. (2011). Environment in the EU27 - Recycling accounted for a quarter of total municipal waste treated in 2009. http://epp.eurostat.ec.europa.eu/cache/ITY_PUBLIC/8-08032011-AP/EN/8-08032011-APEN.PDF. Junho.

Finlay, P. N. (1994). Introducing decision support systems. Oxford, UK Cambridge, Blackwell Publishers.

Ford, A. (2009). Modeling the Environment. Island Press.

Garbelini, S.M. et al.(2011). Pratique Coleta Seletiva. Ministério Público de Goiás.

Gharajedaghi, J. (2006). Systems thinking: managing chaos and complexity. 2 Ed. Elsevier.

Hisatugo, E. e Marçal Jr, O. (2007). Coleta Seletiva e Reciclagem como Instrumentos para Conservação Ambiental: Um Estudo de Caso em Uberlândia - MG. Sociedade \& Natureza, v.19,n.2, páginas 205- 216 . 
IBGE. (2002). Pesquisa Nacional de Saneamento Básico. Instituto Brasileiro de Geografia e Estatística. http://www.ibge.gov.br/home/estatistica/populacao/condicaodevida/pnsb/pnsb. pdf, Junho.

IBGE. (2010). Censo Demográfico 2010. Instituto Brasileiro de Geografia e Estatística, 2010. http://www.ibge.gov.br/home/download/estatistica.shtm, Junho.

Law, A.M., Kelton, W.D. (1991). Simulation Modeling \& Analysis. 2 Ed., McGraw-Hill.

Mansvelt, J. (2010). Green Consumerism: An A-to-Z Guide. SAGE Publications.

Ministério das Cidades - Secretaria Nacional de Saneamento Ambiental. (2010). Sistema Nacional de Informações sobre Saneamento: diagnóstico do manejo de resíduos sólidos urbanos - 2008. MCIDADES-SNSA.

Ministério do Meio Ambiente . (2011). Plano Nacional de Resíduos Sólidos. MMA.

Monteiro, J.H.P. et al. (2001). Manual de Gerenciamento Integrado de Resíduos Sólidos. IBAM - Instituto Brasileiro de Administração Municipal.

Netto, A. (2001). O Desperdício do Lixo. Jornal Zero Hora, 09 de setembro de 2001, Página 32.

O'Leary, P.R. et al.(1999). Decision Maker's Guide to Solid Waste Management. v. 2. Washington DC: U.S. Environmental Protection Agency.

Silva, E.C.P. (2006). O impacto da gestão do tamanho da força policial na taxa de violência em Curitiba: Uma abordagem qualitativa sob o referencial da dinâmica de sistemas. 2006. Dissertação (Mestrado em Engenharia de Produção e Sistemas) - Pontifícia Universidade Católica do Paraná.

Simonetto, E. O., Borenstein, D. (2006). Simulação computacional para auxílio na determinação do escoamento da coleta seletiva de resíduos. III Simpósio Brasileiro de Sistemas de Informação, Curitiba-PR. Anais do III Simpósio Brasileiro de Sistemas de Informação. UNICENP.

Straus, L.M. (2010). Um modelo em dinâmica de sistemas para o ensino superior. 2010. Dissertação (Mestrado em Administração) - Universidade Federal do Rio Grande do Sul.

Sufian, M.A., Bala B.K. (2007). Modeling of urban solid waste management system: The case of Dhaka city. Waste Management, v.27, páginas 858-868.

UNEP - United Nations Environment Programme. (2005). Solid Waste Management. v.1. CalRecovery Inc.

Ventana Systems. (2012). Vensim Simulation Software, http://www.vensim.com . Agosto

Waste Management. (2012). Recycling Facts and Tips. http://sacramento valley. wm.com/recyclingfacts.asp . Agosto. 